\title{
Modelo pedagógico para el desarrollo de competencias en educación superior
}

\section{Celia Carrera Hernández \\ Profesora investigadora \\ Universidad Pedagógica Nacional, Unidad 081}

\section{Resumen}

E

n la Universidad Pedagógica Nacional

Unidad 081 se buscó construir y apli-

car un modelo pedagógico denomina-

do DECOES (Desarrollo de Competencias en

Educación Superior), basado en la teoría de la

elaboración (Reigeluth y Stein 1983, Gil, 2000)

y en el análisis de las aportaciones teóricas so-

bre el término competencias. Se aplicó con un

diseño cuasiexperimental, a fin de conocer su impacto en el desarrollo de la competencia de Intervención Educativa del segundo semestre de la licenciatura en Intervención Educativa. En él participaron un grupo experimental y otro como control. El modelo pedagógico se operó en una guía didáctica. La competencia se evaluó mediante un esquema de pretest y postest. Los datos recabados se analizaron mediante la técnica de análisis de varianza de medidas repetidas empleando el SPSS 15.0. Se encontró que no existen diferencias significativas entre grupo control y experimental en la aplicación del pretest; mientras que en el postest sí existen diferencias significativas entre ambos grupos con $p=0.000$. Se infiere que el modelo pedagógico DECOES tuvo efectos en el grupo experimental al favorecer el logro de la competencia.

Palabras clave: Desarrollo de competencias, modelo pedagógico, aprendizaje, práctica educativa, evaluación. 


\section{Introducción}

La Universidad Pedagógica Nacional diseñó la Licenciatura en Intervención Educativa (LIE), con un modelo curricular basado en competencias que desde el año 2002 se imparte en algunas unidades de UPN en el país, entre ellas la unidad 081 de Chihuahua, Chih.

Sin embargo, se identificaron dificultades en su operación, como la falta de congruencia entre el desarrollo de la docencia y el enfoque por competencias, ya que el ejercicio de la primera no se centra en el aprendizaje, sino en el desarrollo de contenidos que en algunos casos son teóricos; como práctica educativa se analizan textos y se realizan exposiciones por los alumnos; al mismo tiempo la enseñanza está descontextualizada de la realidad del alumno, lo cual dificulta la comprensión de los temas trabajados y disminuye el interés por desarrollar las actividades propuestas.

Con base en lo anterior, surgió la idea de diseñar un modelo pedagógico para el desarrollo de competencias en la educación superior que contemplara principios teóricos fundamentales derivados de diversos estudios psicopedagógicos sobre el aprendizaje, al mismo tiempo que incluyera elementos metodológicos importantes para el docente.

En este artículo se presentan los principios teóricos en que se fundamentan el modelo y los elementos metodológicos que pueden ser considerados como herramientas importantes para que el profesor desarrolle una docencia más congruente con el enfoque por competencias y con los principios del modelo.
Se incluyen los pasos para el desarrollo de una guía didáctica, la forma en que se desarrolló el estudio y los resultados del experimento.

\section{Las competencias}

La forma en que se definen las competencias en el presente trabajo, se articula con el enfoque cognitivo, con carácter socio-constructivo, que da significancia a las competencias necesarias para actuar de manera exitosa en la sociedad como: la cooperación, la solución de problemas y la toma de decisiones (Mulder, 2008). Además en este enfoque se resalta la importancia de la investigación, el diálogo entre maestro y alumno, así como la actuación sobre las prácticas dentro de un contexto.

Arribar a un concepto de competencias resulta muy difícil pues existe una gran variedad de significados debido a la experiencia que se tiene respecto a su aplicación (Gonczi, 1997), a esto se le agrega la poca experiencia en el análisis sobre su traducción en prácticas (Gimeno, 2008). Sin embargo se realiza un esfuerzo por clarificar el uso del término y de traducirlo en implicaciones prácticas.

Es importante mencionar que el concepto de competencias nace desde posiciones básicamente funcionales, por lo que fue aplicado en el ámbito profesional antes que en el educativo, hace referencia a acciones humanas eficientes y a una aplicación del conocimiento (Zabala, 2008). Por ello MacClelland (1973) definió competencia como una forma de evaluar aquello que realmente causa un rendimiento superior en el trabajo. Cuando se 
habla de competencias profesionales se cita a este autor, ya que se centra en la función de la competencia y lo relaciona con la calidad de los resultados del trabajo (Zabala, 2008).

Para Le Boterf (2000) la competencia es la secuencia de acciones que combina varios conocimientos, un esquema operativo transferible a una familia de situaciones. Para este autor, la competencia es la construcción, resultado de combinar recursos cognitivos. Le Boterf (2001) conceptualiza las competencias como el saber actuar dentro de un contexto de trabajo, combinando los recursos necesarios para el logro de un resultado excelente y que es válido en una situación de trabajo, lo cual indica que el dominio de la competencia implica tanto el desempeño del individuo, como el medio y los recursos disponibles para su ejecución a partir de las expectativas que se generan en un ambiente sociocultural determinado. Para este autor, no se trata de un conglomerado de elementos que estructuran la competencia, sino de un saber que no se transmite, donde el centro de la competencia es el alumno que construye la competencia a partir de la secuencia de las actividades de aprendizaje que movilizan los conocimientos especializados (Cano, 2008).

Como lo menciona Zabala (2008) Le Boterf es quien hace referencia a los recursos que son movilizados e integrados en la práctica, al mismo tiempo incorpora el concepto de familia de situaciones, referidas a situaciones -problema conectadas. Este concepto es retomado en el ámbito educativo.

Las definiciones de competencia profesional arriba mencionadas tienen como fin la realización de tareas eficaces, al mismo tiempo estas tareas tienen un contexto de aplicación basado en unas normas, por lo que implican la puesta en práctica bajo un formato de aplicación con una revisión exhaustiva de la calidad en la operación. Lo anterior indica que, en el ámbito laboral, el uso del término competencia es rígido y facilita el establecimiento de diferencias entre los trabajadores.

Por otro lado, en el ámbito educativo las definiciones de competencia se explican desde un paradigma interpretativo ya que se exige del alumno: iniciativa, transferencia del conocimiento e innovación (Cano, 2008), pues el aprendizaje se constituye en un acto complejo que demanda del alumno una actitud de reflexión, crítica, participación y búsqueda de información nueva. Se visualizan tanto factores internos como externos en el proceso y aplicación de la competencia, además de hacer constante referencia al contexto.

Los factores internos se refieren a las características de las personas que las desarrollan a partir del saber — saber-hacer y saber-ser-; los externos se refieren a los materiales que requiere utilizar, a los recursos tecnológicos y a los profesores que son fuente de experiencia y conocimiento indispensable para el nuevo profesionista y, por último, el contexto profesional que incluye los compañeros de trabajo y las normas de desempeño establecidas para su evaluación (Gimeno, 2008).

En el ámbito educativo, el término competencia implica mayor profundidad y extensión y precisa la forma en que se movilizan los componentes de la competencia. Por ello Zabala (2008) expresa que la competencia 
consiste en la intervención ética en los diferentes ámbitos de la vida, mediante acciones en las que se movilizan al mismo tiempo y de manera interrelacionada componentes actitudinales, procedimentales y conceptuales.

Si en una competencia se movilizan conocimientos, procedimientos y actitudes, se refiere a los saberes que ha de tener el alumno; los cuales sirven de referente para saber si ha logrado desarrollar la competencia; estos saberes forman parte de una competencia y hablan al mismo tiempo de su aspecto estructural.

Le Boterf (2000) define la competencia como un saber entrar en acción, lo cual implica saber integrar, movilizar y transferir un conjunto de recursos (conocimientos, saberes, aptitudes y razonamientos) en un contexto dado, a fin de realizar una tarea o de hacer frente a diversos problemas que se presentan.

Este autor hace mención de la movilización de recursos para aplicarlos en un contexto dado, al mismo tiempo que reconoce su utilidad para resolver problemas.

Según De Ketele (1996), la competencia es un conjunto ordenado de capacidades que se ejercen sobre los contenidos de aprendizaje y cuya integración permite resolver los problemas que se plantean dentro de una categoría de situaciones. Se trata de ejecutar una tarea compleja, o un conjunto de tareas más o menos del mismo tipo, dentro de una familia de situaciones.

Perrenoud (2003) define la competencia como una capacidad de actuar eficazmente en una situación de un tipo definido, capacidad que se apoya en los conocimientos, pero que no se reduce a ellos. Para hacer frente, lo mejor posible, a una situación debemos poner en juego y en sinergia varios recursos cognitivos, entre ellos los conocimientos.

Cuando Perrenoud hace referencia a los recursos que el alumno utiliza, los llama cognitivos, pero que no precisamente deberá referirse a conceptualizaciones, sino que incluye el conocimiento de procedimientos.

Para Roeigers (2002) la competencia es la posibilidad que tiene un individuo de movilizar, de manera interiorizada, un conjunto integrado de recursos, con el fin de resolver una familia de situaciones.

Hasta este momento se mantiene la idea de las movilizaciones, recursos, resolver problemas también llamados situaciones-problema.

Según Zabala (2008) la competencia ha de identificar aquello que necesita cualquier persona para dar respuesta a los problemas a los que se enfrentará a lo largo de su vida. Por lo tanto la competencia consistirá en la intervención eficaz en los diferentes ámbitos de la vida, mediante acciones en las que se movilizan al mismo tiempo y de manera interrelacionada, componentes actitudinales, procedimentales y conceptuales.

Zabala (2008) coincide con Roeigers (2007) en que la movilización de recursos es de manera integrada, lo mismo que son utilizados para hacerle frente a situaciones.

Estos elementos comunes permiten deducir que los recursos cognitivos no se aplican de forma separada, sino de manera interrelacionada o integral. Aspectos que se consideran característicos del término. 
Cuando hablamos de competencias hacemos referencia a una dimensión estructural y a otra semántica que permiten, a su vez, identificar características o rasgos comunes que podemos utilizar para definir el término con mayor claridad.

Las características comunes identificadas son: la movilización o el uso de recursos cognitivos, las situaciones-problemas. Entendiéndose por situación al entorno en el que se desarrolla una actividad y tiene que ver con la articulación del contexto y la tarea.

En la vida cotidiana, las situaciones surgen de la misma realidad del alumno con los acontecimientos diarios. Sin embargo, en el contexto escolar, las situaciones son definidas por los alumnos y el docente bajo un plan de acción. La situación se considera significativa, porque le proporciona el deseo de movimiento y le da sentido a lo que aprende, permite contextualizar sus conocimientos, le plantea un desafío y le permite movilizar saberes. Por ello, centrar la atención en las situacionesproblema es importante cuando se trabaja bajo el enfoque por competencias.

El cuadro No. 1 de la página siguiente resume las características de la competencia, según lo revisado en las conceptualizaciones mencionadas.

De estas características, se desprende que el uso del término competencia en los procesos de enseñanza, aprendizaje y evaluación no es mecánico, sino involucra un proceso reflexivo al introducirse en contextos complejos y cambiantes. Por lo que se deduce que el concepto de competencia es holístico, construc- tivista y dentro de una interpretación comprensiva (Gimeno, 2008: 77) ya que, más que conocimientos y habilidades, es el conjunto de dominios o saberes cognitivos, procedimentales y actitudinales, susceptibles de mejora, que el alumno moviliza de manera deliberada en la solución de situaciones-problemas reales cuando interactúa con el contexto del cual forma parte.

La competencia es la intervención eficaz en los diferentes ámbitos de la vida, mediante acciones en las que se movilizan, al mismo tiempo y de manera interrelacionada, recursos cognitivos, que tienen que ver con una actuación eficiente en un contexto determinado. Se agrega el término eficiente, en este caso, cuando la persona trata de dar respuesta a problemas identificados en espacios donde puede intervenir y se desenvuelve adecuadamente en diferentes ámbitos, con personas diferentes en función de sus necesidades, como es el caso del interventor educativo.

El término competencia implica la movilización de recursos cognitivos de forma integrada en la solución de situaciones-problema, con un conjunto de tareas específicas tendientes a tener una actuación eficiente en un contexto determinado.

\section{Teoría de la elaboración}

La Teoría de la Elaboración de Reigeluth (1983) representa una de las aportaciones más significativas al diseño de la instrucción, por lo que se puso la mirada en esta teoría y se pudo reconocer que el conocimiento generado por su aplicación es de gran importancia, 


\section{Cuadro No.1}

\begin{tabular}{|c|c|c|c|c|c|}
\hline \multirow[b]{2}{*}{ Autor } & \multicolumn{5}{|c|}{ Características de la competencia } \\
\hline & $\begin{array}{l}\text { Movilización de } \\
\text { recursos cognitivos }\end{array}$ & $\begin{array}{l}\text { Carácter } \\
\text { interrelacionado }\end{array}$ & $\begin{array}{l}\text { Uso en la solución } \\
\text { de situaciones- } \\
\text { problema }\end{array}$ & Evaluabilidad & Contexto \\
\hline $\begin{array}{l}\text { Le Boterf } \\
(2001)\end{array}$ & & Combinando recursos & & Resultado excelente & $\begin{array}{l}\text { Contexto de } \\
\text { trabajo }\end{array}$ \\
\hline $\begin{array}{l}\text { Le Boterf } \\
\text { (1995) }\end{array}$ & Movilizar recursos & Saber integrar & $\begin{array}{l}\text { Hacer frente a } \\
\text { problemas }\end{array}$ & & $\begin{array}{l}\text { Contexto } \\
\text { dado }\end{array}$ \\
\hline $\begin{array}{l}\text { De Katele } \\
\text { (1996) }\end{array}$ & & $\begin{array}{l}\text { Integración de } \\
\text { capacidades }\end{array}$ & $\begin{array}{l}\text { Resolver } \\
\text { problemas dentro } \\
\text { de una categoría } \\
\text { de situaciones }\end{array}$ & & \\
\hline $\begin{array}{l}\text { Perrenoud } \\
\text { (1998) }\end{array}$ & & $\begin{array}{l}\text { Poner en juego y } \\
\text { en sinergia recursos } \\
\text { cognitivos. }\end{array}$ & $\begin{array}{l}\text { Situación de un } \\
\text { tipo definido. }\end{array}$ & $\begin{array}{l}\text { Capacidad de actuar } \\
\text { eficazmente. Hacer } \\
\text { frente lo mejor } \\
\text { posible. }\end{array}$ & \\
\hline $\begin{array}{l}\text { Roeigers } \\
(2002)\end{array}$ & $\begin{array}{l}\text { Posibilidad de } \\
\text { movilizar un } \\
\text { conjunto de recursos }\end{array}$ & $\begin{array}{l}\text { Forma interrelacionada } \\
\text { e integrada }\end{array}$ & $\begin{array}{l}\text { Fin de resolver } \\
\text { una familia de } \\
\text { situaciones }\end{array}$ & & \\
\hline Zabala (2008) & $\begin{array}{l}\text { Aquello que necesita } \\
\text { y que se constituyen } \\
\text { en acciones que se } \\
\text { movilizan }\end{array}$ & & $\begin{array}{l}\text { Dar respuesta a } \\
\text { problemas }\end{array}$ & Intervención eficaz & $\begin{array}{l}\text { Ámbitos de la } \\
\text { vida }\end{array}$ \\
\hline
\end{tabular}

debido a la forma en que se propone secuenciar los contenidos y abordar la docencia con elementos cognitivos que permiten a profesores y alumnos tener una visión crítica y global sobre el proceso de aprendizaje.

El aspecto más destacado de esta teoría es el intento de organizar la instrucción, a la que se compara con la captación de una escena por el zoom de una cámara. Al comienzo, la toma es de un gran ángulo, después se va especificando en partes, aumentando el detalle y la complejidad de las partes por medio de la aproximación del zoom.

Luego se regresa al panorama general para integrar la información específica con el todo más complejo y revisar la instrucción previa.

La revisión teórica y la síntesis son aspectos importantes que favorecen el aprendizaje, la retención y la transferencia. Esto recuerda el currículo en espiral propuesto por Bruner o la noción de diferenciación progresiva de Ausubel. El modelo general de la teoría de la elaboración se basa en un enfoque de macroenseñanza que incluye varias fases y pasos (Hernández, 2005).

La teoría de la elaboración de Reigeluth y Stein (1983) se fundamenta en el constructivismo y el aprendizaje significativo e integra dos posiciones teóricas:

1. El análisis de contenido que, de acuerdo con Ausubel (1976), el aprendizaje se da cuando el alumno relaciona los conocimientos nuevos con los que ya posee; este proceso implica tres momentos: 1) Conocer el estado final de los conocimientos del alumno al identi- 
ficar los elementos básicos de la estructura lógica, 2) Utilizar un organizador previo (experienciales, analogías) como puente entre el conocimiento nuevo y el previo, y 3) Organización jerárquica de los conceptos obedeciendo a un orden descendente, esto es, que de manera deductiva se va de los conceptos generales a los específicos.

2. El análisis de tareas propuesto por Gagné (1970), quien señala que la jerarquía de aprendizaje debe darse de manera ascendente, avanzando de las tareas más simples a las complejas, incluso de lo concreto a lo abstracto. Para aprender procedimientos complejos es necesario partir de que el estudiante cuente con experiencia.

Ante estas dos posiciones, aparentemente contradictorias, se alza la tercera opción: la síntesis, que se encuentra en la teoría de la elaboración, donde se busca conciliar lo deductivo (Ausubel, 1976) y lo inductivo (Gagné, 1985), lo cual implica ir de lo general a lo específico, a la vez que avanzar de lo simple a lo complejo o de lo concreto a lo abstracto. Por eso, la teoría de la elaboración se presenta como una analogía donde participan una cámara fotográfica, un zoom y una fotografía. La sesión fotográfica inicia con una toma panorámica -general a lo simple- que contiene una vista de gran angular, lo cual permite ver las partes más importantes de la imagen y las relaciones principales entre ellas, pero sin detalles. Mediante el zoom se da un acercamiento (descenso) a una parte determinada de la imagen (detalle/complejo) y se vuelve de nue- vo a la imagen completa (ascenso) vista desde el gran angular (general), y así sucesivamente.

\section{El modelo pedagógico para el desarrollo de competencias}

Con base en la teoría de la elaboración se construyó el modelo pedagógico con principios y rasgos metodológicos, entre los que destacan:

- Análisis previo de la competencia.

- Establecimiento de redes generales y específicas que incluyan conceptos y metodología del curso (uso de epítomes, la Uve de Gowin y el mapa tridimensional).

- Uso de estrategias de enlace hacia la solución de problemas.

- Uso de apoyos y andamios académicocontextuales.

- Interacción del alumno con el contexto.

- Síntesis e integración del conocimiento.

Los rasgos metodológicos del modelo son:

- Epítomes: Recurso metodológico para establecer síntesis de ideas, conceptos y estrategias que pueden utilizarse al inicio del curso o bloque, así como al final de cada uno de ellos.

- Uve de Gowin: Recurso que apoya al docente para relacionar los contenidos conceptuales, procedimentales y actitudinales.

- Mapa de experto: Rasgo que permite contar con una visión general del curso, la tarea, actividades, estrategias y formas de apoyo. 
- Taxonomía de actividades: Actividades que pueden ser desarrolladas y que permiten al alumno transitar desde situaciones individuales hasta discusiones en pequeños grupos para llegar a la construcción individual final.

La taxonomía de actividades es la siguiente:

1. Análisis del epítome general y particulares.

2. Actividades previas.

3. Actividades de búsqueda y análisis de información.

4. Actividades de socialización.

5. Actividades de síntesis.

6. Actividades de ayuda.

\section{El instrumento}

El instrumento utilizado como pretest y postest se diseñó considerando la competencia de intervención educativa a la que hace referencia el curso donde se aplicó el tratamiento, se consideraron las habilidades para solucionar problemas y tomar decisiones que se incluyen en la parte II y III del test. La primera parte se refiere al cambio conceptual.

En la parte II y III se utilizaron casos para identificar conceptos, problemas y proponer soluciones.

Para conocer el nivel de desarrollo de la competencia de acuerdo al test se utilizó una rúbrica (Díaz Barriga, 2006), que ubica a los alumnos en una escala de valoración del 1 al 5 en la que la puntuación 1 especifica no apto, el nivel 2 novato, el nivel 3 aprendiz, el nivel 4 apto y el 5 competente.

\section{Método}

La investigación buscó explicar, mediante un estudio cuasiexperimental, el efecto de la variable independiente (modelo pedagógico DECOES) en el desarrollo de la competencia del curso (variable dependiente), de los alumnos del segundo semestre de la LIE, en el curso intervención educativa. Considerado como diseño cuasiexperimental, no se utilizó la aleatoriedad para seleccionar a los grupos, sino se trabajó con ellos tal como estaban integrados.

El diseño cuasiexperimental (Campbell y Stanley (2001) empleó el pretest y postest en grupo control y grupo experimental y se manipuló deliberadamente la variable independiente para observar su efecto y relación con el desarrollo de la competencia del curso.

El análisis de los datos se realizó de manera inferencial mediante la técnica de medidas repetidas (SPSS, 15.0), la cual proporciona un análisis de varianza, cuando se toma la misma medida varias veces a cada sujeto o grupo, en este caso fue a los grupos control y experimental.

\section{Resultados}

Se encontraron diferencias significativas entre grupo control y experimental después de la aplicación del tratamiento, a partir de la comparación de resultados, considerando los factores inter-sujetos e intra-sujetos. De esta manera, se probó la hipótesis de que existen diferencias significativas en el logro de la competencia entre el grupo control y experimental derivadas del modelo pedagógico. 
Respecto a la parte I del instrumento, el grupo control no presenta diferencia significativa con respecto al grupo experimental en el pretest. Por el contrario, en el postest existe diferencia significativa entre el grupo control y el experimental, esto se atribuye al trabajo con los conceptos abordados a partir del modelo.

Se aprecia también que el grupo control tuvo un ligero avance en la "calificación", del pretest al postest (cuadro 2) en cuanto al cambio conceptual, debido al manejo de información, a las actividades de enseñanza desarrolladas por el profesor y la participación de los alumnos durante las clases. Sin embargo, este avance no es significativo en relación al alcanzado por el grupo experimental, el cual refleja una diferencia amplia en los cambios conceptuales.

En la parte II del instrumento, se encontró que no hay diferencia significativa en cuanto a los resultados del pretest en los grupos control y experimental. Sin embargo, se aprecia una diferencia significativa $(\mathrm{p}=0,000)$ entre los dos grupos en el postest.
De acuerdo con los resultados de la parte III, se encuentra que no hay diferencia significativa entre los grupos control y experimental en el pretest. Esta diferencia sí es significativa en el postest, pues refleja el resultado del tratamiento en el grupo experimental, que se traduce en la experiencia de identificar problemas reales y proponer alternativas de solución; esto permitió resolver el test con mayor facilidad y determinar el bosquejo del proyecto de intervención; mientras que para los alumnos del grupo control resultó de gran dificultad resolver esta parte, pues la única experiencia sobre problemas fue a partir de ejemplos de proyectos de intervención presentados en las exposiciones de algunos equipos (método didáctico privilegiado en este grupo). El nivel de significancia $(p=0,000)$ manifiesta que en esta tercera parte, aún cuando los dos grupos avanzaron, hay diferencias significativas entre ambos.

Finalmente, en las puntuaciones del test integrado se aprecia que no existe diferencia en los resultados del pretest, pero existe una diferencia significativa en el postest entre el grupo control y experimental.

Cuadro No. 2 Medias ( $x$ ) y niveles de significancia ( $p$ )

\begin{tabular}{|c|c|c|c|c|c|c|c|c|c|}
\hline & & \multicolumn{2}{|c|}{ Parte I } & \multicolumn{2}{|c|}{ Parte II } & \multicolumn{2}{|c|}{ Parte III } & \multicolumn{2}{|c|}{ Test integrado } \\
\hline & & Pretest & Postest & Pretest & Postest & Pretest & Postest & Pretest & Postest \\
\hline \multirow{2}{*}{$\begin{array}{l}\text { Grupo de } \\
\text { control }\end{array}$} & $p$ &, 573 & , 000 & ,317 & ,000 & ,058 & ,000 & 191 & ,000 \\
\hline & $\mathrm{x}$ & 36.49 & 47.66 & 43.18 & 59.42 & 26.08 & 37.14 & 35.25 & 48.07 \\
\hline \multirow{2}{*}{$\begin{array}{l}\text { Grupo } \\
\text { experimental }\end{array}$} & $p$ &, 573 &, 000 & ,317 & ,000 & ,058 & ,000 & 191 & ,000 \\
\hline & $x$ & 37.69 & 87.68 & 38.85 & 88.04 & 29.75 & 95.96 & 35.43 & 90.56 \\
\hline
\end{tabular}




\section{Conclusiones}

De los resultados anteriores se infiere que el tratamiento tuvo efectos positivos en el grupo experimental al favorecer el logro de la competencia del curso y sobre todo el desarrollo de habilidades como el análisis y la solución de problemas. Sobre la tipología trabajada y sus fundamentos, se recomienda su uso en la construcción de guías didácticas para el desarrollo de competencias. Se considera que el modelo pedagógico DECOEs puede ser aplicado en otros cursos, sobre todo relacionados con la formación en intervención educativa y de otras profesiones, ya que permite a los estudiantes relacionar los conocimientos desarrollados en la escuela con las experiencias que provee el medio, con el fin de incidir en el desarrollo de diferentes grupos sociales. Se prevé que el desarrollo de competencias en la educación superior mejoraría al establecer cambios en sus prácticas educativas, transitando de una docencia centrada en los contenidos a una docencia centrada en el aprendizaje, en la cual, a partir del análisis, identificación e intervención en la solución de problemas, el estudiante pueda tener cambios en los conceptos que inicialmente posee.

\section{Bibliografía}

Ausubel D. (1976). Psicología educativa. Un punto de vista cognitivo. México: Trillas.

Ausubel D., Novak J., y Hanesian H. (2006) Psicología educativa. Un punto de vista cognoscitivo. Trillas. México.

Campbell y Stanley (2001). Diseños experimentales y cuasiexperimentales de la investigación social. Argentina: Amorrortu.

Cano García, M. E. (2008) La evaluación por competencias en la educación superior. Universidad de Barcelona. Revista electrónica de currículum y formación del profesorado. España.

De Ketele (1996) L' evaluation des acquis scolaires:quoi? Pourquoi? Pourquoi?. En Revue tunisienne des sciences de' éducation No. 23. Brussels: Boeck.
Díaz Barriga, F. (2006). Enseñanza situada: vínculo entre la escuela y la vida. México: Mc Graw Hill.

Gagné, R. M. (1970). Las condiciones del aprendizaje. Madrid; Aguilar.

(1985). The conditions of learning. New York. Holt.

Gil Julia, et al. (2000). Macrosecuencia instruccional de óptica siguiendo la teoría de la elaboración. España: Universidad de Extremadura.

Gimeno Sacristán, J. (2008) Educar por competencias, ¿Qué hay de nuevo?. Editorial Morata. Barcelona, España.

Gonczi A. y Athanasou J. (2000) Instrumentación de la educación basada en competencias. Perspectivas de la teoría y la práctica en Australia, en Competencia laboral y educación basada en normas de competencia de Antonio Argüelles (comp.), México, Limusa, SEP, CNCCL y CONALEP.

Gonczi, A. (1997). Problemas asociados con la implementación de la educación basada en la competencia: de lo atomístico a lo holístico, en Formación basada en competencia laboral (México, Conocer-OIT/ Cinterfor).

(2000) Perspectivas internacionales de la Educación Basada en Competencias. Conferencia en la Universidad Tecnológica de Sydney.

Hernández Hernández, P. (2005) Psicología de la educación. Corrientes actuales y teorías aplicadas. Trillas. México.

Le Boterf, G. (1995) Ingeniería de las competencias. Gestión. España.

(2000) Construire les competentes individuelles et collectives. París: Editions d'Organisation.

(2001) Ingeniería de Las Competencias. Ediciones Gestión 2000. Barcelona, España.

McClelland, D. (1973). Testing for Competence Rather Than for Intelligence. American Psychologist, 28, 1-14.

Mulder, M. (2008) El concepto de competencia en el desarrollo de la educación y formación profesional en algunos Estados miembros de la UE: un análisis crítico. Profesorado. Revista de Currículum y Formación de Profesorado [en línea] 2008, 12 (Sin mes): [fecha de consulta: 12 de julio de 2010] en: $<$ http://redalyc.uaemex.mx/src/inicio/ArtPdfRed. jsp?iCve $=56712875007>$

Perrenoud, P. (2003) Construir competencias desde la escuela. $2^{\mathrm{a}}$ Edición. Editorial Sáenz.

Reigeluth y Stein (1983). The Elaboration Theory of Instruction". En C. M. Reigeluth, Instructional design: theories and models: an overwiev of their current status. Hilldsdale, New Yersey.

Roegiers, X. (2002). Une pe'dagogie de l'inte'gration [Integrative pedagogy]. Brussels: De Boeck.

(2007) Pedagogía de la integración. Colección Ider. Francia.

SPSS. Modelos avanzados. Versión 15. Chicago: SPSS Inc.

Zabala A. y Laia A.(2008) 11 ideas clave. Cómo aprender y enseñar competencias. Grao. Barcelona, España. 